\title{
Detection of genomic regions associated malformations in newborn piglets: a machine-learning approach
}

\author{
Siroj Bakoev ${ }^{\text {Corresp., } 1,2}$, Aleksei Traspov ${ }^{1,2}$, Lyubov Getmantseva ${ }^{\text {Corresp., } 1}{ }^{1}$, Anna Belous ${ }^{1}$, Tatiana Karpushkina ${ }^{1}$, Olga \\ Kostyunina ${ }^{1}$, Alexander Usatov ${ }^{3}$, Tatiana V Tatarinova ${ }^{4,5,6,7}$ \\ ${ }^{1}$ Federal Research Center for Animal Husbandry named after Academy Member LK. Ernst, Dubrovitsy, Russia \\ 2 Centre for Strategic Planning and Management of Biomedical Health Risks, Moscow, Russia \\ 3 South Federal University, Rostov-on-Don, Russia \\ 4 Department of Biology, University of La Verne, La Verne, California, United States \\ 5 Institute for Information Transmission Problems, Russian Academy of Sciences, Moscow, Russia \\ 6 Vavilov Institute for General Genetics, Moscow, Russia \\ 7 School of Fundamental Biology and Biotechnology, Siberian Federal University, Krasnoyarsk, Russia \\ Corresponding Authors: Siroj Bakoev, Lyubov Getmantseva \\ Email address: siroj1@yandex.ru, ilonaluba@mail.ru
}

Background. A significant proportion of perinatal losses in pigs occurs due to congenital malformations. The purpose of this study is the identification of genomic loci associated with fetal malformations in piglets. Methods. The malformations were divided into two groups: associated with limb defects (piglet splay leg) and associated with other congenital anomalies found in newborn piglets. 148 Landrace and 170 Large White piglets were selected for the study. A genome-wide association study based on the gradient boosting machine algorithm was performed to identify markers associated with congenital anomalies and piglet splay leg. Results. 49 SNPs (23 SNPs in Landrace pigs and 26 SNPs in Large White) were associated with congenital anomalies, 22 of which were localized in genes. A total of 156 SNPs (28 SNPs in Landrace; 128 in Large White) were identified for piglet splay leg, of which 79 SNPs were localized in genes. We have demonstrated that the gradient boosting machine algorithm can identify SNPs and their combinations associated with significant selection indicators of studied malformations and productive characteristics. Data availability. Genotyping and phenotyping data are available at http://www.compubioverne.group/data-and-software/ 
Siroj Bakoev ${ }^{1,2^{*}}$, Aleksei Traspov ${ }^{1,2}$, Lyubov Getmantsevaa ${ }^{1, *}$, Anna Belous ${ }^{1}$, Tatiana Karpushkina ${ }^{1}$, Olga Kostyunina ${ }^{1}$, Alexander Usatov ${ }^{3}$, Tatiana Tatarinova ${ }^{4-7}$

${ }^{1}$ Federal Research Center for Animal Husbandry named after Academy Member LK. Ernst, Dubrovitsy, Russia

${ }^{2}$ Centre for Strategic Planning and Management of Biomedical Health Risks, Moscow, Russia

${ }^{3}$ South Federal University, Rostov-on-Don, Russia

${ }^{4}$ Department of Biology, University of La Verne, La Verne, California, United States of America

${ }^{5}$ Institute for Information Transmission Problems, Russian Academy of Sciences, Moscow, Russia

${ }^{6}$ Vavilov Institute for General Genetics, Moscow, Russia

7 School of Fundamental Biology and Biotechnology; Siberian Federal University, Krasnoyarsk, Russia

\section{* Corresponding Authors:}

Siroj Bakoev ${ }^{1,2}$

${ }^{1}$ Federal Research Center for Animal Husbandry named after Academy Member LK. Ernst, Dubrovitsy, Russia

${ }^{2}$ Centre for Strategic Planning and Management of Biomedical Health Risksdisabled, Moscow, Russia

Email address: siroj1@yandex.ru

Lyubov Getmantseva ${ }^{1}$

${ }^{1}$ Federal Research Center for Animal Husbandry named after Academy Member LK. Ernst, Dubrovitsy, Russia

Email address: ilonaluba@mail.ru

\section{Abstract}

Background. A significant proportion of perinatal losses in pigs occurs due to congenital malformations. The purpose of this study is the identification of genomic loci associated with fetal malformations in piglets.

Methods. The malformations were divided into two groups: associated with limb defects (piglet splay leg) and associated with other congenital anomalies found in newborn piglets. 148 Landrace and 170 Large White piglets were selected for the study. A genome-wide association 
37 study based on the gradient boosting machine algorithm was performed to identify markers

38 associated with congenital anomalies and piglet splay leg.

39 Results. 49 SNPs (23 SNPs in Landrace pigs and 26 SNPs in Large White) were associated with 40 congenital anomalies, 22 of which were localized in genes. A total of 156 SNPs (28 SNPs in 41 Landrace; 128 in Large White) were identified for piglet splay leg, of which 79 SNPs were 42 localized in genes. We have demonstrated that the gradient boosting machine algorithm can 43 identify SNPs and their combinations associated with significant selection indicators of studied 44 malformations and productive characteristics.

45 Data availability. Genotyping and phenotyping data are available at

46 http://www.compubioverne.group/data-and-software/ 


\section{Introduction}

48

49

50

51

52

53

54

55

56

57

58

59

60

61

62

63

64

65

66

67

68

69

70

71

72

73

74

75

76

77

78

79

80

81

82

83

84

85

86

The mortality of piglets represents a severe loss for the pig farming industry (Pig Progress, 2014; Walters, 2016). The losses predominantly occur during the last days of pregnancy, delivery, and the first three days of life (Woollen, 1993). Many factors contribute to the loss of piglets: the age of sows and their maternal qualities, the birth duration, the litter size, the parity number, farm management, and other factors (Olsson, Botermans \& Englund, 2018). Pigs have more frequent congenital malformations than any other domestic animal (Woollen, 1993), and congenital malformations account for a significant proportion of perinatal losses.

The profitable production of pigs requires that a farm consistently produces healthy piglets (Staarvik et al., 2019). However, the piglet mortality rate is positively correlated with the number of piglets born (Olsson, Botermans \& Englund, 2018), making it a limiting factor for farm growth. Therefore, identifying and addressing the causes of congenital malformations in pigs is essential for pig farming.

Congenital diseases often pose a diagnostic problem, having unknown etiology, pathology, and prognosis. This is also true beyond animal husbandry: up to $79 \%$ of human congenital diseases have unknown etiology (Papatsiros \& Others, 2012).

The frequency of occurrence of specific malformations varies from farm to farm. Breeds with small populations have smaller genetic diversity and are prone to more defects. The most common defect, affecting between $2.3 \%$ and $5.0 \%$ of all newborn piglets, is the piglet splay leg (PSL) (Papatsiros \& Others, 2012). PSL is a condition when a newborn piglet cannot hold its hindlimbs together (in severe cases, the forelimbs are also affected) and cannot stand or walk after birth (Partlow et al., 1993) and may, therefore, starve. There are also cases of inguinal, scrotal, and umbilical hernia, cryptorchidism, and atresia. The prevalence of this condition is between $2.3 \%$ and $5.0 \%$.

Genetic factors are believed to play an essential role in the development of congenital malformations in piglets. Studies found genetic drivers of PSL (Hao et al., 2017) and cleft palate (which is a lethal anomaly in pigs) describing chromosomal imbalances in affected offspring (Grahofer et al., 2019). All affected offspring were carriers of partial trisomy of chromosome 14, including the FGFR2 gene associated with various dominant hereditary syndromes of craniofacial dysostosis in humans. Hao et al. (Hao et al., 2017) presented seven significant SNPs and four genes related to muscle development, glycogen metabolism, and mitochondrial dynamics, identified as potential candidate genes for PSL. However, only limited research aimed at finding SNPs and candidate genes associated with congenital malformations in pigs has been conducted (Bermingham et al., 2014; Ji et al., 2016; Bakoev et al., 2020).

When analyzing extensive genomic data with traditional parametric models, the problem of a small number of observations and a large number of predictive variables (typically, tens or even hundreds of thousands of SNPs are interrogated in thousands or, sometimes, hundreds of samples) is challenging to solve due to the high dimension or highly correlated data structure (Li et al., 2018). Traditional GWAS analysis focuses on a univariate hypothesis and assumes the presence of independent explanatory variables. These methods suffer significantly from a lack of power and

Peer] reviewing PDF | (2020:12:56522:2:1:NEW 12 May 2021) 
87 accuracy when dealing with the complexity of multiple interactions or correlations between

88

89

90

91

92

93

94

95

96

97

98

99

100

101

102

103

104

105

106

107

108

109

110

111

112

113

114

115

116

117

118

119

120

121

122

123

predictors (e.g., SNP-SNP and SNP- covariate interactions) (Lettre, Lange \& Hirschhorn, 2007; Zheng et al., 2007; So \& Sham, 2011; Adams, Bello \& Dumancas, 2015). Machine learning approaches, and Gradient Boosting Machines (GBM), in particular, are suitable for such complex problems (Li et al., 2018). Various machine learning applications were developed to build a nonparametric regression or classification model from data. One strategy is to build a model from theoretical considerations and then define its parameters based on the observed data. The main idea behind the GBM algorithm is to create new baseline learners that are maximally correlated with the negative gradient of the loss function associated with the entire ensemble. There is a wide choice of existing loss functions that can be adjusted depending on the specific task at hand. This flexibility makes GBMs highly customizable for any specific data-driven task. It introduces much freedom in model design, making it a matter of patience since it takes trial and error to select the most appropriate loss function.

We have conducted a pilot GWAS to identify potential genomic regions and genes associated with congenital diseases and anomalies in newborn piglets. We used two common pig breeds for our study: Landrace and Large White. The Landrace pigs originated from the free-breeding, nonpedigreed stock of swine, the regional landrace native to Denmark. The Large White pig was produced in the $18^{\text {th }}$ century by crossing the large indigenous white pig of North England with the smaller, fatter, white Chinese pig. These breeds have different genetics but share the problems with congenital malformations. We use the gradient boosting machine (GBM) approach since it is flexible and relatively easy to implement. Moreover, GBM has demonstrated considerable success in practical applications and various machine learning and data extraction tasks (Bissacco, Yang \& Soatto, 2007; Hutchinson, Liu \& Dietterich, 2011; Pittman \& Brown, 2011; Johnson \& Zhang, 2014). We have evaluated the applicability of GSM for searching for genomic areas and genes associated with congenital anomalies and compared its performance to standard approaches.

\section{Materials \& Methods}

\section{Samples}

We have an ongoing collaboration with pig breeding farms in Russia. The tissue samples were collected at the same breeding farm with a closed breeding system by the farm employees over the last decade. Ear clipping and phenotyping were performed following the standard EU monitoring procedures and guidelines $(2010 / 63 / \mathrm{EU})$ at the farm. The piglets that were assessed as healthy were kept alive, and the affected piglets were culled, and the ear clipping was performed post-mortem. Per 2010/63/EU guidelines, ear clipping and pig slaughter do not require a separate IRB approval or anesthesia. We have collected tissue samples, and phenotypic information on all available piglets at the pig farm determined to have birth defects by specially trained farm employees. We have also collected samples from healthy piglets from the same farm to form control groups. 
124

125

126

127

128

129

130

131

132

133

134

135

136

137

138

139

140

141

142

143

144

145

146

147

148

149

150

151

152

153

154

155

156

157

158

159

160

161

Among the 148 Landrace piglets, 75 were healthy, 27 had piglet splay legs, and 46 had other congenital anomalies. Among the 170 Large White piglets, 108 were healthy, 38 had piglet splay legs, and 32 had other congenital anomalies. All sampled piglets were female.

Genomic DNA was extracted from the tissue samples using a set of DNA-Extran-2 reagents (NPF Syntol LLC, Russia) per the manufacturer's instructions. The quantity, quality, and integrity of DNA were evaluated using a Qubit 2.0 desktop fluorometer ("Invitrogen"/"Life Technologies", USA) and a NanoDrop8000 spectrophotometer ("ThermoFisherScientific", USA).

The animals were genotyped using the GeneSeek Genomic Profiler (GGP) BeadChip for Porcine HD (Illumina, Neogen), comprising 68,516 SNPs. According to the recommendations, genotype quality control was performed using Plink 1.9 (Marees et al., 2018). After excluding SNPs with a missing sample frequency $>2 \%$ and a Hardy-Weinberg equilibrium (HWE) p-value $<10^{-7}, 45,218$ SNPs for the Landrace pigs and 44,324 SNPs for the Large White pigs were retained for further analysis.

Significant SNPs were identified separately for the two breeds and two congenital disabilities.

\section{Data preprocessing and function selection}

The malformations detected in newborn piglets were divided into two groups by assessed qualities: leg defects (piglet splay leg, PSL) and other congenital anomalies (CA). The analysis was carried out using a case-control design (Table 1). The dependent variable was binary: presence ("Yes") or absence ("No") of the condition, as evaluated by professional pig breeders.

For the gene-phenotype association analysis, a manifestation of congenital anomalies in piglets and PSL genotypes was used as a predictor in the model. We used the Plink 1.9 0/1/2 encoding for genotypes (Purcell et al., 2007). The data were randomly divided into training (80\%) and testing $(20 \%)$ sets. In each iteration of the training, hyperparameters were set up using a search within a 10 -fold cross-checking structure on a random $80 \%$ of the training set. The most effective hyperparameters were used to train the model and tested on the test set. The gradient boosting machine (GBM) algorithm was used to select the most significant predictors among SNPs.

All scripts were written in $\mathrm{R}$ and executed on the $\mathrm{H} 2 \mathrm{O}$ platform (Click et al., 2016).

\section{Population Structure Analysis}

To analyze the population structure, we used two related techniques: Singular value decomposition (SVD) using the script from (Porto-Neto et al., 2013) and Principal component analysis (PCA), using command princomp in R. Plots of top 10 principal components were done in R. Admixture analysis was performed using the ADMIXTURE algorithm (Alexander, Novembre \& Lange, 2009) for varying the number of components between 2 and 9. Crossvalidation errors reported by the ADMIXTURE and GPS algorithms (Elhaik et al., 2014) were used to identify the optimal number of components.

GPS algorithm was used for predicting the provenance of all genotyped individuals. If no geographic information is supplied for the reference samples, GPS identifies the closest reference 
162 populations to the tested sample. If geographic information is available, GPS also finds a global

163

164

165

166

167

168

169

170

171

172

173

174

175

176

177

178

179

180

181

182

183

184

185

186

187

188

189

190

191

192

193

194

195

196

197

198

199

position where the individuals with the genotype closest to the tested one live. If there is no geographic information, GPS can find the nearest individuals among the reference dataset. GPS is not suitable to analyze recently admixed individuals. GPS works by calculating the Euclidean distance between the sample's admixture proportions and the reference dataset.

We also used the genomic relationship matrix (GRM), which is calculated by the formula (VanRaden, 2008) $\mathrm{G}=\frac{\mathrm{ZZ}}{2 \sum_{\mathrm{i}}^{N} \mathrm{p}_{\mathrm{i}}\left(1-\mathrm{p}_{\mathrm{i}}\right)}$, where $\mathrm{p}_{\mathrm{i}}$ is the alternative allele frequency in the $\mathrm{i}^{\text {th }}$ locus. Calculation of matrix $Z$ requires several steps. At first, matrix $P$, containing $\left(2 \mathrm{p}_{\mathrm{i}}-1\right)$ in each cell, is calculated. It has dimension $L x N$, where $L$ is the number of SNPs, and $N$ is the number of animals. Next, genotype matrix $M$ is calculated, where genotypes are coded as $-1 / 0 / 1(-1$ is homozygous in alternative allele, 0 is heterozygous, and +1 is homozygous in reference allele). Matrix $\mathrm{Z}$ is the difference between $P$ and $M$, and $\mathrm{Z} '$ is the transposition of matrix $\mathrm{Z}$.

SVD is a valuable tool for characterizing the genetic structure to detect and extract small signals even if the data is noisy (Berrar, Dubitzky \& Granzow, 2007). We performed the SVD and visualized the relationships between populations using R. SNPRelate (Zheng et al., 2012) was used to perform principal component analysis (PCA), Identity-By-State (IBS) analysis, relationship inference, and hierarchical clustering.

\section{Machine Learning Analysis}

GBM can provide an accurate estimate of the response variable by creating new base learners maximally correlated with the negative gradient of the loss function associated with the entire ensemble. Using the classical quadratic error loss function (L2 norm), the training procedure produces a sequence of error-fitting steps. AUC ("Area Under the receiver operating characteristic Curve") is used to assess classifier performances: better classifiers have larger values of AUC. AUCPR (Precision-Recall) - Logistic Loss - is a logistic loss function that penalizes the classifier's confidence in an incorrect response; Gini index was used for evaluating the quality of classification, Mean Per-Class Error is the average value for a class error.

We have selected the following parameters of GBM to minimize the validation error: the number of trees $($ ntree $=1000)$, learning rate (learn_rate $\left.=10^{-3}\right)$, maximum tree depth (max_depth $=10$ ), and number of cross-checks (nfolds $=10$ ).

We stratified the samples by breed and type of defect. All samples were randomly divided into training $(80 \%)$ and testing $(20 \%)$ sets. In each iteration of the training, hyperparameters were set up using a search within a 10 -fold cross-checking structure on a random $80 \%$ of the training set. The most effective hyperparameters were used to train the model and tested on the test set. GBM algorithm was used to select the most "important" predictors among SNPs. GBM approach uses relative importance to rank the SNPs. At each split in each tree, GBM computes the mean squared error (MSE) for regression (the tree split criterion). GBM averages the MSE improvement (decrease) made by each variable across all the trees that the variable is used. The variables with the largest average decrease in MSE are considered the most important. The values of the relative

Peer) reviewing PDF | (2020:12:56522:2:1:NEW 12 May 2021) 
200 importance of variables vary from 0 to 1 ("Relative variable importance chart for CART®) 201 Regression"; Natekin \& Knoll, 2013). Because GBM is a collection of individual decision trees, 202 the relative importance value is a measure of the critical variable for evaluating the target variable. 203 We considered all SNPs with positive relative importance to be significant.

204 GBM algorithm can potentially identify too many SNP with positive relative importance when 205 complex traits are analyzed. To reduce the false-positive rate, functional annotation of SNPs is 206 conducted, intersection with other methods is determined, and only the highest scoring SNPs are 207 considered.

208 SNPs with positive relative importance were used to generate a list of associated SNPs; their 209 positions and corresponding genes were examined with the Ensembl! browser (Sus scrofa 210 11.1)(“Ensembl genome browser 102”). The functional annotation was performed using the 211 Panther database ("PANTHER - Gene List Analysis") and the Variant Effect Predictor (VEP) tool 212 (http://uswest.ensembl.org/Sus_scrofa/Tools/VEP/) (McLaren et al., 2016; Khimsuriya et al., 213 2019). To find the GWAS studies for the human orthologs, we used the EBI GWAS Catalog 214 (https://www.ebi.ac.uk/gwas/home) and a manual search in the literature for data on their 215 associations with any human or animal traits.

216

217

218

219

220

221

222

223

224

225

226

227

228

229

230

231

232

233

234

235

\section{Association Analysis with rrBLUP and Plink}

We compared the performance of GBM with rrBLUP (Endelman, 2011) and Plink (Purcell et al., 2007) approaches.

We used the function GWAS from the rrBLUP R package using flags min.maf $=0.05$ and $P 3 D$ $=$ FALSE (equivalent to EMMA with REML) and selected SNPs with marker scores $-\log _{10} \mathrm{p}>2$, which is equivalent to the condition of $\mathrm{p}$-value $<10^{-2}$.

Plink was used with the following options: --all-pheno --allow-no-sex --bfile $<\ldots>-$-model -pheno $<. .>$. This command conducts five tests: The tests offered here are (in addition to the basic allelic test): basic allelic test, Cochran-Armitage trend test, Genotypic test, Dominant gene action test, and Recessive gene action test. We chose SNPs with asymptotic $p$-value $<10^{-2}$ in at least one of these five tests.

\section{IRB Approval}

All procedures were performed under the guidelines approved by the LK. Ernst Federal Research Center for Animal Husbandry (Russia) and with the rules for conducting laboratory research (tests) in the implementation of veterinary control (supervision) approved by Council Decision Eurasian Economic Commission No. 80 (November 10, 2017).

\section{Results}

We have conducted admixture analysis of both breeds for $\mathrm{K}=2 \ldots 11$. Both breeds appear to be genetically diverse. Both Admixture and PCA (Supp. Fig. 1) plots showed that LA and LW pigs 
236

237

238

239

240

241

242

243

244

245

246

247

248

249

250

251

252

253

254

255

256

257

258

259

260

261

262

263

264

265

266

267

268

269

270

271

272

273

274

belong to two different sample groups. However, for all K (Figure 1), the admixture profiles of Large White pigs are a mosaic of almost all components, while a smaller number of admixture components represents the Landraces. Therefore, the sampled Landrace pigs were genetically more homogeneous than the Large White pigs. To determine the optimal number of components, we have analyzed cross-validation error and accuracy of clustering.

As a function of the number of admixture components, the cross-validation error drops to a plateau value at K=4. Next, we used NbClust (Charrad M., Ghazzali N., Boiteau V., Niknafs A., 2014) kmeans procedure in $\mathrm{R}$ to partition the samples using 26 different scores to define the optimal number of clusters. The number of clusters for $\mathrm{K}>3$ was between 6 and 8 .

\section{Figure 1. Admixture plots for Landrace and Large White pigs, K=2...11.}

GPS (Elhaik et al., 2014) leave-one-out analysis validated the clustering assignment correctness. For $\mathrm{K}=2$ and 3 , nearly $10 \%$ of the samples were misclassified (the rest was placed to correct clusters). For the higher values of K, GPS was placing $100 \%$ of samples into correct clusters. The fraction of affected animals per cluster ranged from $11 \%$ to $42 \%$ for splay leg phenotype and $13 \%$ to $83 \%$ for other congenital anomalies. For example, for $\mathrm{K}=11$, in cluster LA_2, all six piglets were affected; one had a splay leg and five other congenital anomalies. In cluster LW_3, having 23 members, three piglets were born with splay legs, and no other anomalies were detected.

We have calculated Pearson's correlation coefficient between piglets' status and values of admixture components. For example, for $\mathrm{K}=18$, in both breeds, the presence of congenital anomalies is negatively correlated with the admixture component $\mathrm{K} 8$ (LW: $\mathrm{r}=-0.26$, t-test $\mathrm{p}$ value $=0.0002$ ). This effect is especially pronounced in Large White piglets: $3 \%$ of piglets with $\mathrm{K} 8>0.3$ ( 1 out of 38 ) have congenital defects, as compared to $31 \%$ of piglets with $\mathrm{K} 8<0.3$ (29 out of 94). Cluster LW_7, where K8 is the main ancestry, is the healthiest. Congenital anomalies in Large White piglets are positively correlated with the admixture component $\mathrm{K} 5$ ( $\mathrm{r}=0.21$, $\mathrm{t}$-test $\mathrm{p}$ value $=0.007$ ). $41 \%$ of piglets with $\mathrm{K} 5>0.3$ ( 15 out of 37 ) have congenital defects, as compared to $16 \%$ of piglets with $\mathrm{K} 5<0.3$ (15 out of 95 ). Therefore, it appears that certain pig lines are more susceptible to the development of congenital anomalies.

Figure 2. Diversity of samples analysis. Hierarchical clustering (Figure 2, A), PCA analysis (Figure 2, B) showed the separation of Landrace and Large White pigs into two distinct groups. Relatedness (Figure 2, C) and IBS (Figure 2, D) plots show that Large White samples mainly were from unrelated individuals, while the Landrace samples range from unrelated to first-degree relatives. Clustering (A) and IBS (D) plots also showed population stratification within the Large White pigs: the samples could be separated into two main clusters.

Peer] reviewing PDF | (2020:12:56522:2:1:NEW 12 May 2021) 
$275 \quad \mathrm{~F}_{\mathrm{st}}$ between Landrace and Large White pigs was estimated by the method of Weir \& Cockerham 276 (Weir and Cockerham, 1984) to be 0.165. Hierarchical clustering (Figure 2, A), PCA analysis 277 (Figure 2, B) showed the separation of Landrace and Large White pigs into two distinct groups. 278 Note that the PCA plot had the arch and horseshoe effects for Large White pigs. These effects are 279 common in ecological and population studies when the sampling units cover a long ecological 280 gradient. Those samples at each end of the gradient have few species in common. These artifacts 281 produce visually beautiful but false patterns that are not present in the original data. Using the 282 SNPRelate package in R (Zheng et al., 2012), we have calculated relatedness (Figure 2, C) and 283 IBS (Figure 2, D). Within- and between-breed relatedness have been inferred by the IBD using 284 the Plink method of moments (Purcell et al., 2007). We have calculated the Cotterman coefficients 285 of relatedness K0 and K1 (Cotterman, 1940). These plots show that Large White samples mainly 286 were from unrelated individuals, while the Landrace samples range from unrelated to first-degree 287 288 289 290 291

292

293 relatives. Clustering (A) and IBS (D) plots also showed population stratification within the Large White pigs: the samples could be separated into two main clusters.

Next, we used the rrBLUP (Endelman, 2011) R package and Plink (Chang et al., 2015) to find significant SNPs associated with the splay leg and congenital malformations phenotypes separately for the Large White and the Landrace breeds. Plink performs five tests: Basic Allelic test, Cochran-Armitage trend test, Genotypic test, Dominant gene action test, and Recessive gene action test.

Using the p-value cut-off of $10^{-5}$, there were no significant SNPs in Landrace pigs identified by Plink (p-value $<10^{-5}$ in at least one of the five Plink tests) and also by rrBLUP (Figure 3). In Large White pigs, one SNP (MARC0062453 a.k.a. rs80912121) was found significant by two Plink tests for piglet splay leg (its rrBLUP p-value $=1.334 \times 10^{-5}$, hence, it nearly misses the cutoff). It is an intron variant for CRISP3 (cysteine-rich secretory protein 3) on chromosome 7. For other congenital anomalies of Large White pigs, rrBLUP has identified two SNPs, intergenic variant ALGA0086405 and downstream gene variant WU_10.2_13_216912741, the first of each was significant in four out of five tests, according to Plink. SNP ALGA0086405 (rs81454026) is located on Chromosome 15 position 92,733,414. There are three genes near this SNP: GULP, TFPI, and CALCRL. Gene GULP PTB (domain-containing engulfment adaptor 1) is located on Chromosome 15: 92,838,715-93,251,019. Gene TFPI (tissue factor pathway inhibitor) is located on Chromosome 15: 92,345,075-92,409,905. Gene CALCRL (calcitonin receptor-like receptor) is located on Chromosome 15: 92,233,841-92,332,363. These genes may be associated with the disease since, due to artificial selection and small effective population size in pigs, the LD in pigs is much larger than in humans (Badke et al., 2012).

SNP WU_10.2_13_216912741 (located downstream of a lncRNA located on Chromosome 13: 207,036,621-207,042,808 reverse strand) was not significant according to Plink. Plink has identified four SNPs (ALGA0097461, ASGA0009945, WU_10.2_2_80100147, and WU_10.2_6_139448200), that were not significant according to rrBLUP. ALGA0097461 is in an intron of a lncRNA on Chromosome 18: 23,230,190-23,234,168, forward strand. ASGA0009945 is an intron variant of gene growth arrest-specific 2 gene GAS2 on chromosome 2: 36,713,106- 
315

316

317

318

319

320

321

322

323

324

325

326

327

328

329

330

331

332

333

334

335

336

337

338

339

340

341

342

343

344

345

346

347

348

349

350

351

352

353

$36,840,407$ reverse strand. WU_10.2_2_80100147 is an intron variant of TBC1 domain family member 9B, located on Chromosome 2:78548370 (forward strand). WU_10.2_6_139448200 is an intergenic variant on Chromosome 6:151776796 (forward strand).

\section{Figure 3. Manhattan plots for Splay Leg (A,B,E,F) and Other Congenital malformations $(C, D, G, H)$ phenotypes in Landrace $(A, B, C, D)$ and Large White $(E, F, G, H)$ piglets, rrBLUP and Plink. Since Plink performs five tests for each SNP, the average negative logarithm of the p-value is plotted for Plink.}

Next, we used the GBM approach (see Table 2, Table 3, Supp. Table 5, and Supp. Table 6. A complete list of GBM SNPs is in Supp Table 7). GBM has identified 205 variants combined from separate analyses of two breeds and two conditions. The Ensembl Variant Effect Prediction tool classified the SNPs into the following categories: intron variant: 71\%; non coding transcript variant: $9 \%$; intergenic variant: $8 \%$; downstream gene variant: 5\%; upstream gene variant: 4\%; 3prime UTR variant: $2 \%$; non coding transcript exon variant: $1 \%$; synonymous variant: $1 \%$. To compare the GBM and Plink performance, we have calculated the confusion matrices (Supp. Table 8). GBM was more accurate than Plink in labeling the affected piglets as affected, but this came at the cost of a higher false-positive rate. After training and testing the models, the primary performance statistics were calculated (Table 2).

GBM has found 49 SNPs (23 SNPs in LA pigs and 26 SNPs in LW) linked with congenital anomalies. Twenty-two of these SNPs were located in genes (Table 3). In total, 156 significant SNPs were identified for the splay leg condition. In Landrace pigs, twenty-eight SNPs were identified; fifteen were located in genes (Supp. Table 5). In Large White pigs, 128 SNPs were identified (63 of the SNPs were in fifteen genes, Supp Table 6).

The overlap between GBM, Plink (selecting SNPs significant in at least one of the five Plink tests), and rrBLUP identified SNPs is shown in Supp. Figures 2 and 3. Only five SNPs were detected by all three methods: ALGA0075476, MARC0030657, WU_10.2_6_138715609, CASI0008977, and ALGA0021723 (Figure 3).

ALGA0075476, significant in Landraces for splay leg, is on the 14, position 12790416 in the intron of homeobox-containing gene 1, HMBOX1. According to the EBI GWAS catalog, SNPs in this gene's human ortholog are associated with obesity-related traits (https://www.ebi.ac.uk/gwas/variants/rs2221894).

MARC0030657 (also a significant marker in Landraces for the splay leg) is on chromosome 1, position 112210717. This SNP is located in the Intron 5-6 of lncRNA ENSSSCG00000048340. In general, lncRNAs serve as molecular signals to regulate transcription.

WU_10.2_6_138715609 (significant in Large White pigs for the splay leg) is an intergenic variant on chromosome 6, position 150780123. Two more intergenic variants (CASI0008977 at chr2:107190210 and ALGA0021723 chr3:129009157) were identified as significant in Landrace pigs for the splay leg.

Peer) reviewing PDF | (2020:12:56522:2:1:NEW 12 May 2021) 
354

355

356

357

358

359

360

361

362

363

364

365

366

367

368

369

370

371

372

373

374

375

376

377

378

379

380

381

382

383

384

385

386

387

388

389

390

391

392

393

SNP WU_10.2_18_48204887 was identified as significant by GBM for the splay leg in Large White pigs, for other congenital anomalies in Landrace pigs by Plink and rrBLUP. WU_10.2_18_48204887 (on chromosome 18, position 43798925) is in the intron of gene TRIL (TLA4 interactor with leucine-rich repeats). In humans, SNPs in this region are associated with anomalies in the urogenital system.

The reason for the small number of SNPs in the overlap between the three methods is in the approaches used by the programs: the first two programs use parametric methods (negatively affected by small sample size and a large number of SNPs), while in the GBM algorithm, a nonparametric approach is taken.

In Landraces, one of the identified genes (ACSL3, Acyl-CoA synthetase long-chain family member 3) encoded a metabolite interconversion enzyme from the long-chain fatty acid coenzymes A-ligases. All members of this family convert free long-chain acids into esters but differ in tissue-specific expression. Complex fatty acyl-COA esters are substrates for numerous fatty acid metabolic pathways, including mitochondrial $\beta$-oxidation and phospholipids, and triacylglycerol synthesis. Changes in ACSL enzymes can contribute to various physiological processes, including apoptosis, gene transcription, signaling, and vulnerability to oxidative stress (Johnson et al., 2012).

SNP rs344559304 in BTB16 gene (zinc finger and BTB domain-containing 16 as PLZF and ZFP145) received the top score in PSL in Large While pigs. The protein it encodes belongs to the Krüppel-like family of zinc finger proteins. It has been previously shown that ZBTB16 acts as an essential transcriptional repressor or activator (Kolesnichenko \& Vogt, 2011) and participates in diverse biological processes (Dick \& Doulatov, 2009), spermatogenesis (Buaas et al., 2004; Costoya et al., 2004), the formation of hind limbs (Barna et al., 2000), and the apoptosis of cells (Cheung et al., 2010). Impressive results were obtained in studies of the effect of inactivated ZBTB16 on the formation of the skeletal structures of the leg (Wei et al., 2018): ZFP145 -/- mice showed morphological defects affecting the hindlimb's skeletal structures, whereas the forelimb's skeletal defects occurred with lower frequency. Also, ZBTB16 was shown to regulate the expression of the HOX and BMP genes (Wei et al., 2018), influencing leg defect formation.

For PSL in LA pigs, the top SNP was rs81225364, inside an extended non-coding RNA region. LncRNAs participate in many biological processes, such as the regulation of epigenetic modifications (Roberts, Morris \& Weinberg, 2014), embryo development (Zhang et al., 2014), and the development of skeletal muscle (Shi et al., 2020).

We have identified SNP rs327546322, located in the UBAC2 gene (ubiquitin-associated domain-containing protein 2), to be of the highest significance for the congenital anomalies in Landrace pigs. Polymorphisms in the UBAC2 gene are associated with a genetic predisposition to Behcet's disease in humans (Sawalha et al., 2011; Yamazoe et al., 2017), a rare disorder causing blood vessel inflammation throughout the body: in joints, vascular system, lungs, gastrointestinal tract, central nervous system, and epididymis (Kaklamani, Vaiopoulos \& Kaklamanis, 1998). The symptoms include recurrent eye inflammation, oral and genital sores, and skin lesions. No information on polymorphism of the UBAC2 gene in pigs is available in the literature; however, 
394 based on the association of the gene with Behcet's disease and its manifestation in humans, the

395

396

397

398

399

400

401

402

403

404

405

406

407

408

409

410

411

412

413

414

415

416

417

418

419

420

421

422

423

424

425

426

427

428

429

430

431

432

UBAC2 gene is a promising candidate for further investigation of congenital anomalies in pigs.

For congenital anomalies in LW pigs, the top-scoring SNP is rs81374120 in the AUTS2 gene. AUTS2 is one of 10 genes with the highest intron RNA score in the fetal brain (Sultana et al., 2002; Bedogni et al., 2010; Oksenberg \& Ahituv, 2013). (Ameur et al., 2011) showed that alternative splicing programs control the expression of intronic RNAs in the fetal brain. In recent years, the role of the AUTS2 gene in developing autism spectrum disorders has been extensively studied in humans. Also, variants of this gene are associated with brain malformations and congenital disabilities (Beunders et al., 2013). Therefore, it can be hypothesized that the identified SNP rs81374120 in the AUTS2 gene in pigs is associated with nervous system development disorders and leads to congenital anomalies in piglets.

\section{Discussion}

Currently, assessing genetic load is one of the primary problems in pig farming. Various anomalies affect pig breeding's economic efficiency, and the search for genetic factors associated with their manifestation is an urgent task for researchers. It is vital to simultaneously reduce the number of carriers of congenital defects in the Sus scrofa population and improve productivity indicators. It is not possible to solve these problems using classical breeding without using modern genomic selection approaches.

The traditional strategy of culling piglets with phenotypic defects is effective in only a small part of hereditary pathologies caused by dominant or sex-linked genes. However, most Sus scrofa genetic diseases have a recessive or polygenic type of inheritance; therefore, these culling methods are insufficient.

Therefore, the task of finding new methods to identify animals that carry hereditary pathologies is so essential. Nowadays, congenital anomalies cause significant economic losses in the pork industry. The development of genomic technology has given new meaning to the search for solutions.

When analyzing large genomic datasets using conventional parametric models, small observations and large numbers of predictive variables present an obstacle. This problem is challenging to the high dimensionality or strongly correlated data structure (Chen \& Ishwaran, 2012). GWAS analysis, based on traditional statistical methods, focuses on a one-dimensional hypothesis and assumes independent explanatory variables; these methods suffer significantly from a lack of power and accuracy when dealing with the complexity of multiple interactions or correlations between predictors (e.g., SNP-SNP and SNP-covariate interactions) (Lettre, Lange \& Hirschhorn, 2007; Zheng et al., 2007; So \& Sham, 2011; Chen \& Ishwaran, 2012; Adams, Bello \& Dumancas, 2015).

The etiology and pathogenesis of the splay leg are complex and still poorly understood. Histomorphological studies have described PSL as myofibrillar hypoplasia, but this condition has also been detected in clinically normal piglets (Ducatelle et al., 1986). Ultrastructural analysis has

Peer) reviewing PDF | (2020:12:56522:2:1:NEW 12 May 2021) 
433 shown that piglets with PSL have higher muscle glycogen content than normal piglets (Antalíková, 434 Horák \& Matolín, 1996). Maak et al. (Maak et al., 2009) compared gene expression in posterior 435 leg muscles (M. adductores, M. gracilis, M. sartorius) from diseased and healthy piglets. As a 436 result, they proposed four genes (SQSTM1, SSRP1, DDIT4, and MAF) related to splay legs in 437 piglets. Hao et al. (Hao et al., 2017), in a study on 185 animals from four populations (Yorkshire, 438 Duroc, Landrace, and crossbred Yorkshire and Landrace), identified four genes (HOMER1 and 439 JMY on SSC2, ITGA1 on SSC16, and RAB32 on SSC1) associated with muscle development, 440 metabolism, and mitochondrial dynamics that were proposed as candidate genes for PSL.

441

442

443

444

445

446

447

448

449

450

451

452

453

454

455

456

457

458

459

460

461

462

463

464

465

466

467

468

469

Our work has identified 79 genes that could be considered candidate genes for the splay leg. Even though the genes identified by Hao et al. (Hao et al., 2017) were not identified in our work (neither by plink or rrBLUP analysis), it may be noted that in LW pigs, seven of the identified genes (EXT2, NDUFA10, MTMR12, HS6ST3, DBT, ACOX1, CHST11) belong to the protein class "metabolite interconversion enzyme" (PC00262). Metabolic changes have been documented in multifactorial diseases in humans (DeBerardinis \& Thompson, 2012). Metabolism is carried out through enzymatically catalyzed biochemical reactions and involves the mutual conversion of small molecules (metabolites) that play a crucial role in various cellular functions, from energy production to complex biosynthesis macromolecules (Pey et al., 2013). The EXT2 gene (Exostosin Glycosyltransferase 2) encodes one of the glycosyltransferases involved in the chain extension stage of heparan sulfate biosynthesis. EXT2 gene polymorphism is associated with humans with exostosis (or osteoma, benign growth of new bone on top of the existing bone) (Wuyts et al., 1998).

\section{Conclusions}

Our pilot study showed that machine learning approaches could identify genomic loci associated with congenital malformations in piglets. We have identified lists of SNPs and candidate genes associated with congenital anomalies and piglet splay leg for Large White and Landrace pigs. We have also highlighted the need to study farm animals to determine population characteristics and identify genotypes associated with significant selection indicators of malformations and productive qualities.

The small sample size used here is a limitation of our pilot study. We will extend this approach to larger datasets since it is vital for ensuring efficient and ethical handling of farm animals.

\section{Funding}

This research was supported by the RSF Project No. 18-76-00034 (Identification of genome regions related to malformations in newborn piglets); the Russian Foundation for Basic Research 19-016-00068 A (Machine learning-methods for detection of genomic regions and genes affecting piglet splay leg); the State task of the Ministry of science and higher education 04452021-0008 (Genotyping of piglets using GeneSeek Genomic Profiler (GGP) BeadChip for Porcine HD).

Peer) reviewing PDF | (2020:12:56522:2:1:NEW 12 May 2021) 
470

471

472

473

474

475

476

477

478

479

480

481

482

483

484

485

486

487

488

489

490

491

492

493

494

\section{References}

Adams LJ, Bello G, Dumancas GG. 2015. Development and Application of a Genetic Algorithm for Variable Optimization and Predictive Modeling of Five-Year Mortality Using Questionnaire Data. Bioinformatics and biology insights 9:31-41.

Alexander DH, Novembre J, Lange K. 2009. Fast model-based estimation of ancestry in unrelated individuals. Genome research 19:1655-1664.

Ameur A, Zaghlool A, Halvardson J, Wetterbom A, Gyllensten U, Cavelier L, Feuk L. 2011. Total RNA sequencing reveals nascent transcription and widespread co-transcriptional splicing in the human brain. Nature structural \& molecular biology 18:1435-1440.

Antalíková L, Horák V, Matolín S. 1996. Ultrastructural demonstration of glucose-6-phosphatase activity and glycogen in skeletal muscles of newborn piglets with the splayleg syndrome. Reproduction, nutrition, development 36:205-212.

Bakoev S, Getmantseva L, Kolosova M, Kostyunina O, Chartier DR, Tatarinova TV. 2020. PigLeg: prediction of swine phenotype using machine learning. PeerJ 8:e8764.

Barna M, Hawe N, Niswander L, Pandolfi PP. 2000. Plzf regulates limb and axial skeletal patterning. Nature genetics 25:166-172.

Bedogni F, Hodge RD, Nelson BR, Frederick EA, Shiba N, Daza RA, Hevner RF. 2010. Autism susceptibility candidate 2 (Auts2) encodes a nuclear protein expressed in developing brain regions implicated in autism neuropathology. Gene expression patterns: GEP 10:915.

Bermingham ML, Bishop SC, Woolliams JA, Pong-Wong R, Allen AR, McBride SH, Ryder JJ, Wright DM, Skuce RA, McDowell SW, Glass EJ. 2014. Genome-wide association study 

551.

497 498

Berrar DP, Dubitzky W, Granzow M. 2007. A Practical Approach to Microarray Data Analysis. Springer Science \& Business Media.

Beunders G, Voorhoeve E, Golzio C, Pardo LM, Rosenfeld JA, Talkowski ME, Simonic I, Lionel AC, Vergult S, Pyatt RE, van de Kamp J, Nieuwint A, Weiss MM, Rizzu P, Verwer LENI, van Spaendonk RML, Shen Y, Wu B-L, Yu T, Yu Y, Chiang C, Gusella JF, Lindgren AM, Morton CC, van Binsbergen E, Bulk S, van Rossem E, Vanakker O, Armstrong R, Park S-M, Greenhalgh L, Maye U, Neill NJ, Abbott KM, Sell S, Ladda R, Farber DM, Bader PI, Cushing T, Drautz JM, Konczal L, Nash P, de Los Reyes E, Carter MT, Hopkins E, Marshall CR, Osborne LR, Gripp KW, Thrush DL, Hashimoto S, Gastier-Foster JM, Astbury C, Ylstra B, Meijers-Heijboer H, Posthuma D, Menten B, Mortier G, Scherer SW, Eichler EE, Girirajan S, Katsanis N, Groffen AJ, Sistermans EA. 2013. Exonic deletions in AUTS2 cause a syndromic form of intellectual disability and suggest a critical role for the C terminus. American journal of human genetics 92:210220.

Bissacco A, Yang M, Soatto S. 2007. Fast Human Pose Estimation using Appearance and Motion via Multi-Dimensional Boosting Regression. In: 2007 IEEE Conference on Computer Vision and Pattern Recognition. 1-8.

Buaas FW, William Buaas F, Kirsh AL, Sharma M, McLean DJ, Morris JL, Griswold MD, de Rooij DG, Braun RE. 2004. Plzf is required in adult male germ cells for stem cell selfrenewal. Nature Genetics 36:647-652. DOI: 10.1038/ng1366.

Chen X, Ishwaran H. 2012. Random forests for genomic data analysis. Genomics 99:323-329. 
518 Cheung M, Pei J, Pei Y, Jhanwar SC, Pass HI, Testa JR. 2010. The promyelocytic leukemia 519 zinc-finger gene, PLZF, is frequently downregulated in malignant mesothelioma cells and 520 contributes to cell survival. Oncogene 29:1633-1640.

521 Click C, Malohlava M, Candel A, Roark H, Parmar V. 2016. Gradient Boosted Models with 522 H2O. Publ. H2O Ai Inc.

523 Costoya JA, Hobbs RM, Barna M, Cattoretti G, Manova K, Sukhwani M, Orwig KE, 524 Wolgemuth DJ, Pandolfi PP. 2004. Essential role of Plzf in maintenance of

525

526 527 528 529

Elhaik E, Tatarinova T, Chebotarev D, Piras IS, Maria Calò C, De Montis A, Atzori M, Marini

Ducatelle R, Maenhout D, Coussement W, Hoorens JK. 1986. Spontaneous and experimental myofibrillar hypoplasia and its relation to splayleg in newborn pigs. Journal of

Dick JE, Doulatov S. 2009. The Role of PLZF in Human Myeloid Development. Annals of the New York Academy of Sciences 1176:150-153.

DeBerardinis RJ, Thompson CB. 2012. Cellular metabolism and disease: what do metabolic outliers teach us? Cell 148:1132-1144. comparative pathology 96:433-445.

(

Ehaik E, Tatarinova T, Chebotarev D, Piras IS, Maria Calo C, De Montis A, Atzori M, Marini

34
M, Tofanelli S, Francalacci P, Pagani L, Tyler-Smith C, Xue Y, Cucca F, Schurr TG, Gaieski JB, Melendez C, Vilar MG, Owings AC, Gómez R, Fujita R, Santos FR, Comas D, Balanovsky O, Balanovska E, Zalloua P, Soodyall H, Pitchappan R, Ganeshprasad A, Hammer M, Matisoo-Smith L, Wells RS, Genographic Consortium. 2014. Geographic population structure analysis of worldwide human populations infers their biogeographical origins. Nature communications 5:3513.

Endelman JB. 2011. Ridge Regression and Other Kernels for Genomic Selection with R Package 
rrBLUP. The plant genome 4:250-255.

542 Ensembl genome browser 102. Available at https://www.ensembl.org/index.html (accessed $543 \quad$ December 27, 2020).

544 Grahofer A, Letko A, Häfliger IM, Jagannathan V, Ducos A, Richard O, Peter V, Nathues H, 545 Drögemüller C. 2019. Chromosomal imbalance in pigs showing a syndromic form of $546 \quad$ cleft palate. BMC genomics 20:349.

547 Hao X, Plastow G, Zhang C, Xu S, Hu Z, Yang T, Wang K, Yang H, Yin X, Liu S, Wang Z, 548 Wang Z, Zhang S. 2017. Genome-wide association study identifies candidate genes for 549 piglet splay leg syndrome in different populations. BMC genetics 18:64.

550 Hutchinson RA, Liu L-P, Dietterich TG. 2011. Incorporating Boosted Regression Trees into $551 \quad$ Ecological Latent Variable Models. In: AAAI. Citeseer, 1343-1348.

552 Ji HY, Yang B, Zhang ZY, Ouyang J, Yang M, Zhang XF, Zhang WC, Su Y, Zhao KW, Xiao 553

554 SJ, Yan XM, Ren J, Huang LS. 2016. A genome-wide association analysis for susceptibility of pigs to enterotoxigenic Escherichia coli F41. Animal: an international

555 journal of animal bioscience 10:1602-1608.

556 Johnson CO, Lemaitre RN, Fahrenbruch CE, Hesselson S, Sotoodehnia N, McKnight B, Rice 557 KM, Kwok P-Y, Siscovick DS, Rea TD. 2012. Common variation in fatty acid genes and resuscitation from sudden cardiac arrest. Circulation. Cardiovascular genetics 5:422-

559 429.

560

561 IEEE Transactions on Pattern Analysis and Machine Intelligence 36:942-954. DOI:

562 10.1109/tpami.2013.159.

563 Kaklamani VG, Vaiopoulos G, Kaklamanis PG. 1998. Behçet's Disease. Seminars in arthritis 
and rheumatism 27:197-217.

565 Khimsuriya Y, Vaniyawala S, Banaganapalli B, Khan M, Elango R, Shaik NA. 2019. Finding a 566 Needle in a Haystack: Variant Effect Predictor (VEP) Prioritizes Disease Causative 567 Variants from Millions of Neutral Ones. In: Shaik NA, Hakeem KR, Banaganapalli B, 568 Elango R eds. Essentials of Bioinformatics, Volume II: In Silico Life Sciences: Medicine. Cham: Springer International Publishing, 85-104.

570

571

572

573

574

575

576

577

578

579

580

581

582

583

584

585

586

Kolesnichenko M, Vogt PK. 2011. Understanding PLZF: two transcriptional targets, REDD1 and smooth muscle $\alpha$-actin, define new questions in growth control, senescence, selfrenewal and tumor suppression. Cell cycle 10:771-775.

Lettre G, Lange C, Hirschhorn JN. 2007. Genetic model testing and statistical power in population-based association studies of quantitative traits. Genetic epidemiology 31:358362.

Maak S, Boettcher D, Tetens J, Wensch-Dorendorf M, Nürnberg G, Wimmers K, Swalve HH, Thaller G. 2009. Identification of candidate genes for congenital splay leg in piglets by alternative analysis of DNA microarray data. International journal of biological sciences $5: 331-337$.

Marees AT, de Kluiver H, Stringer S, Vorspan F, Curis E, Marie-Claire C, Derks EM. 2018. A tutorial on conducting genome-wide association studies: Quality control and statistical analysis. International journal of methods in psychiatric research 27:e1608.

McLaren W, Gil L, Hunt SE, Riat HS, Ritchie GRS, Thormann A, Flicek P, Cunningham F. 2016. The Ensembl Variant Effect Predictor. Genome biology 17:122.

Natekin A, Knoll A. 2013. Gradient boosting machines, a tutorial. Frontiers in neurorobotics 7:21. 
587 Oksenberg N, Ahituv N. 2013. The role of AUTS2 in neurodevelopment and human evolution.

$588 \quad$ Trends in genetics: TIG 29:600-608.

589 Olsson A-C, Botermans J, Englund J-E. 2018. Piglet mortality \textendash A parallel comparison

590 between loose-housed and temporarily confined farrowing sows in the same herd. Acta

591 Agriculturae Scandinavica, Section A Itextemdash Animal Science 68:52-62.

592 PANTHER - Gene List Analysis. Available at http://www.pantherdb.org/(accessed December $593 \quad 27,2020)$.

594 Papatsiros VG, Others. 2012. The splay leg syndrome in piglets: A Review. American Journal of $595 \quad$ Animal and Veterinary Sciences 7:80-83.

596 Partlow GD, Fisher KR, Page PD, MacMillan K, Walker AF. 1993. Prevalence and types of birth 597 defects in Ontario swine determined by mail survey. Canadian journal of veterinary 598 research $=$ Revue canadienne de recherche veterinaire 57:67-73.

599 Pey J, Tobalina L, de Cisneros JPJ, Planes FJ. 2013. A network-based approach for predicting 600 key enzymes explaining metabolite abundance alterations in a disease phenotype. $B M C$

601 systems biology 7:62.

602 Pittman SJ, Brown KA. 2011. Multi-scale approach for predicting fish species distributions 603 across coral reef seascapes. PloS one 6:e20583.

604 Porto-Neto LR, Lee SH, Lee HK, Gondro C. 2013. Detection of signatures of selection using Fst. 605 Methods in molecular biology 1019:423-436.

606 Purcell S, Neale B, Todd-Brown K, Thomas L, Ferreira MAR, Bender D, Maller J, Sklar P, de 607 Bakker PIW, Daly MJ, Sham PC. 2007. PLINK: a tool set for whole-genome association 608 and population-based linkage analyses. American journal of human genetics 81:559-575.

609 Relative variable importance chart for CART® Regression. 
610 Roberts TC, Morris KV, Weinberg MS. 2014. Perspectives on the mechanism of transcriptional 611 regulation by long non-coding RNAs. Epigenetics: official journal of the DNA

612

613

614

615

616

617

618

619

620

621

622

623

624

625

626

627

628

629

630

631

632 Methylation Society 9:13-20.

Sawalha AH, Hughes T, Nadig A, Yılmaz V, Aksu K, Keser G, Cefle A, Yazıcı A, Ergen A, Alarcón-Riquelme ME, Salvarani C, Casali B, Direskeneli H, Saruhan-Direskeneli G. 2011. A putative functional variant within the UBAC2 gene is associated with increased risk of Behçet's disease. Arthritis and rheumatism 63:3607-3612.

Shi T, Hu W, Hou H, Zhao Z, Shang M, Zhang L. 2020. Identification and Comparative Analysis of Long Non-Coding RNA in the Skeletal Muscle of Two Dezhou Donkey Strains. Genes 11. DOI: 10.3390/genes11050508.

So H-C, Sham PC. 2011. Robust association tests under different genetic models, allowing for binary or quantitative traits and covariates. Behavior genetics 41:768-775.

Staarvik T, Framstad T, Heggelund M, Brynjulvsrud Fremgaarden S, Kielland C. 2019. Bloodglucose levels in newborn piglets and the associations between blood-glucose levels, intrauterine growth restriction and pre-weaning mortality. Porcine health management $5: 22$.

Sultana R, Yu C-E, Yu J, Munson J, Chen D, Hua W, Estes A, Cortes F, de la Barra F, Yu D, Haider ST, Trask BJ, Green ED, Raskind WH, Disteche CM, Wijsman E, Dawson G, Storm DR, Schellenberg GD, Villacres EC. 2002. Identification of a novel gene on chromosome 7q11.2 interrupted by a translocation breakpoint in a pair of autistic twins. Genomics 80:129-134.

VanRaden PM. 2008. Efficient methods to compute genomic predictions. Journal of dairy science 91:4414-4423. 
633 Wei S, Zhang M, Zheng Y, Yan P. 2018. ZBTB16 Overexpression Enhances White

634

635

636

637

638

639

640

641

642

643

644

645

646

647

648

649

650

651

Adipogenesis and Induces Brown-Like Adipocyte Formation of Bovine White Intramuscular Preadipocytes. Cellular physiology and biochemistry: international journal of experimental cellular physiology, biochemistry, and pharmacology 48:2528-2538.

Woollen NE. 1993. Congenital diseases and abnormalities of pigs. The Veterinary clinics of North America. Food animal practice 9:163-181.

Wuyts W, Van Hul W, De Boulle K, Hendrickx J, Bakker E, Vanhoenacker F, Mollica F, Lüdecke HJ, Sayli BS, Pazzaglia UE, Mortier G, Hamel B, Conrad EU, Matsushita M, Raskind WH, Willems PJ. 1998. Mutations in the EXT1 and EXT2 genes in hereditary multiple exostoses. American journal of human genetics 62:346-354.

Yamazoe K, Meguro A, Takeuchi M, Shibuya E, Ohno S, Mizuki N. 2017. Comprehensive analysis of the association between UBAC2 polymorphisms and Behçet's disease in a Japanese population. Scientific reports 7:742.

Zhang K, Huang K, Luo Y, Li S. 2014. Identification and functional analysis of long non-coding RNAs in mouse cleavage stage embryonic development based on single cell transcriptome data. BMC genomics 15:845.

Zheng G, Joo J, Lin J-P, Stylianou M, Waclawiw MA, Geller NL. 2007. Robust ranks of true associations in genome-wide case-control association studies. BMC proceedings 1 Suppl $1: \mathrm{S} 165$ 


\section{Table $\mathbf{1}$ (on next page)}

Frequencies of congenital anomalies in Landrace and Large White breeds. "

Yes"- defect present, "No"- defect absent. 
1

2

\begin{tabular}{lcc}
\hline Breed & Yes & No \\
\hline Landrace & Congenital Anomalies & 56 \\
Large White & 46 & 106 \\
& 32 & \\
Landrace & Piglet Splay Leg & 75 \\
Large White & 27 & 108 \\
\hline
\end{tabular}

Table 1. Frequencies of congenital disabilities in breeds. "Yes"- defect present, "No"defect absent. 


\section{Table 2 (on next page)}

Model performance statistics i index

Models are assessed using Log-Loss, Mean Per-Class Error, AUC, AUCPR, Gini index. 
1 Table 2. Differentially enriched PANTHER pathways identified by the $F_{S T}$ method (LW_Old 2 vs. $L W_{-}$New)

\begin{tabular}{|c|c|c|c|c|}
\hline \# & Name & Expected & $\begin{array}{c}\text { Fold } \\
\text { Enrichment }\end{array}$ & $\begin{array}{l}\text { raw } P- \\
\text { value }\end{array}$ \\
\hline 1 & Synaptic vesicle trafficking & 0.07 & 14.95 & $6.67 \mathrm{E}-02$ \\
\hline 2 & $\begin{array}{l}\text { Biosynthesis of purines de novo (De novo purine } \\
\text { biosynthesis) }\end{array}$ & 0.08 & 11.88 & $8.27 \mathrm{E}-02$ \\
\hline 3 & General transcription regulation & 0.09 & 10.78 & $9.06 \mathrm{E}-02$ \\
\hline 4 & $\begin{array}{l}\text { Signaling pathway of muscarinic acetylcholine } \\
\text { receptor } 1 \text { and } 3 \text { signaling pathway) }\end{array}$ & 0.14 & 7.24 & $1.31 \mathrm{E}-01$ \\
\hline 5 & $\begin{array}{l}\text { Transcription regulation by the bZIP transcription } \\
\text { factor }\end{array}$ & 0.14 & 7.13 & $1.33 \mathrm{E}-01$ \\
\hline 6 & Alzheimer disease-amyloid secretase pathway & 0.15 & 6.53 & $1.44 \mathrm{E}-01$ \\
\hline 7 & $\mathrm{~T}$ cell activation & 0.20 & 4.98 & $1.84 \mathrm{E}-01$ \\
\hline 8 & PDGF signaling pathway & 0.31 & 3.26 & $2.66 \mathrm{E}-01$ \\
\hline 9 & $\begin{array}{l}\text { Path to the receptors of gonadotropin-releasing } \\
\text { hormone (Gonadotropin-releasing hormone } \\
\text { receptor or pathway) }\end{array}$ & 0.54 & 1.87 & $4.17 \mathrm{E}-01$ \\
\hline
\end{tabular}

3 


\section{Table 3(on next page)}

Significant SNPs for Congenital Anomalies in Landrace and Large White

The list of SNPs with functional annotation using VEP. 
1 Table 3: Differentially enriched PANTHER pathways identified by the $F_{S T}$ method (old vs new)

\begin{tabular}{|l|l|l|c|c|c|}
\hline No & & Name & Expected & $\begin{array}{c}\text { Fold } \\
\text { Enrichment }\end{array}$ & $\begin{array}{c}\text { raw P- } \\
\text { value }\end{array}$ \\
\hline 1 & LW_1 & Wnt signaling pathway & 0.08 & 11.49 & $8.20 \mathrm{E}-02$ \\
\hline 2 & LW_2 & Unclassified & 6.16 & 1.14 & $1.00 \mathrm{E}-00$ \\
\hline 3 & LW_3 & Unclassified & 3.52 & 1.14 & $1.00 \mathrm{E}-00$ \\
\hline 4 & LW_4 & General transcription regulation & 0.03 & 28.68 & $3.52 \mathrm{E}-02$ \\
\hline 5 & LW_4 & Transcription regulation by the bZIP & 0.05 & 19.12 & $5.19 \mathrm{E}-02$ \\
\hline 6 & LW_4 & Parkinson disease & & & \\
\hline 7 & LW_4 & Cadherin signaling pathway & 0.11 & 9.16 & 1.04 E-01 \\
\hline 8 & LW_4 & Huntington disease & 0.14 & 7.12 & 1.32 E-01 \\
\hline 9 & LW_4 & Wnt signaling pathway & 0.24 & 4.13 & 2.17 E-01 \\
\hline
\end{tabular}


Figure 1

Admixture profiles for $\mathrm{K}=2 . .11$

Admixture plots for $\mathrm{K}=2 \ldots 11$ for Landrace (LA) and Large White (LW) pigs.

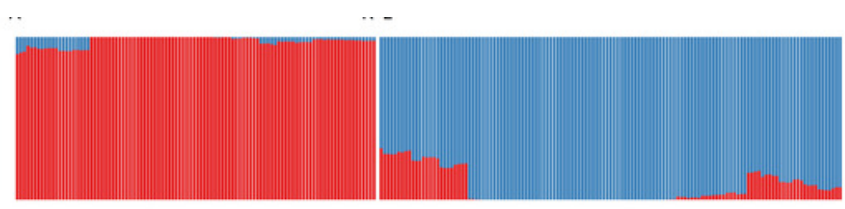

Landrace

Large White

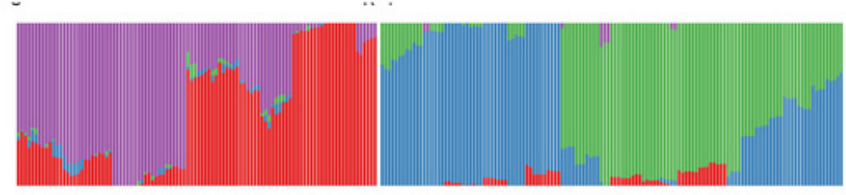

Landrace

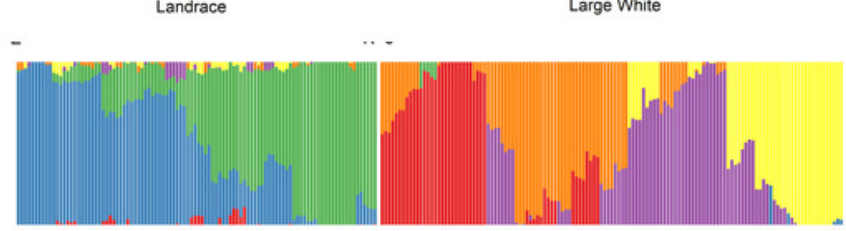

Landrace

Large White

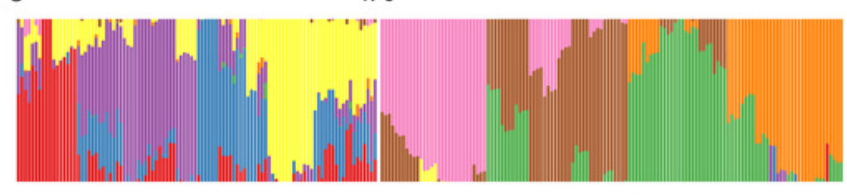

Landrace

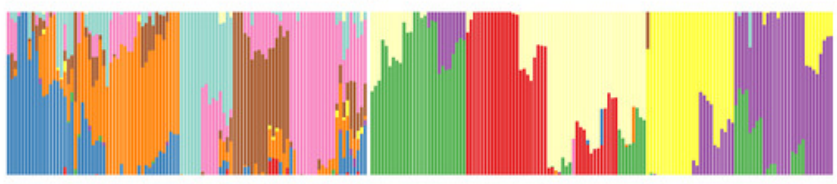

Landrace

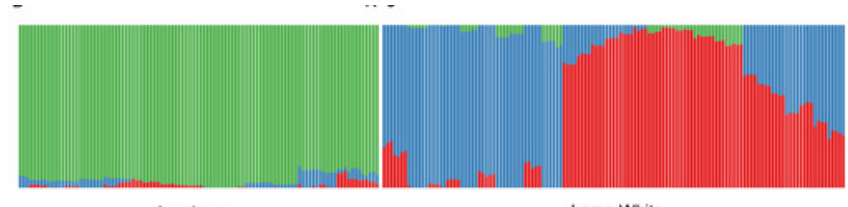

Landrace

Large White

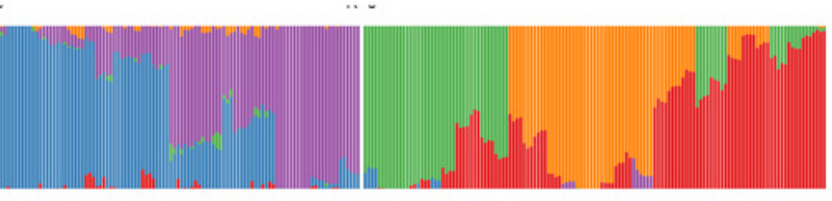

Landrace

Large White

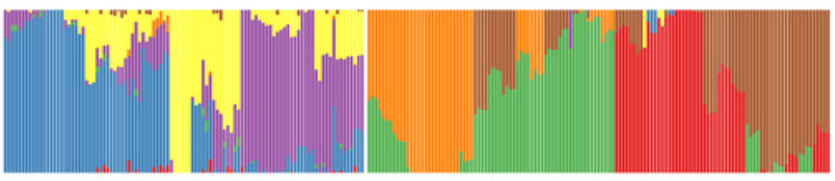

Landrace

Large White

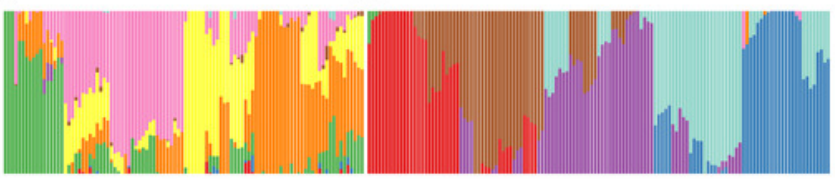

Landrace

Large White

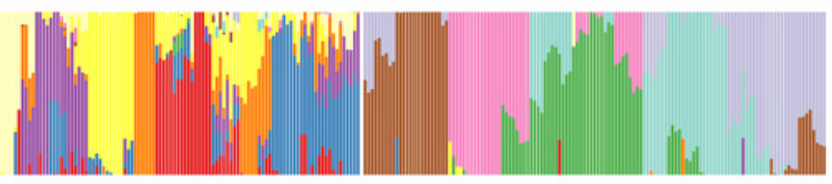

Landrace

Large White 


\section{Figure 2}

Diversity of samples analysis.

Figure 2. Hierarchical clustering (Figure 2, A), PCA analysis (Figure 2, B) showed the separation of Landrace and Large White pigs into two distinct groups. Relatedness (Figure 2, C) and IBS (Figure 2, D) plots show that Large White samples mainly were from unrelated individuals, while the Landrace samples range from unrelated to first-degree relatives. Clustering (A) and IBS (D) plots also showed population stratification within the Large White pigs: the samples could be separated into two main clusters.

A
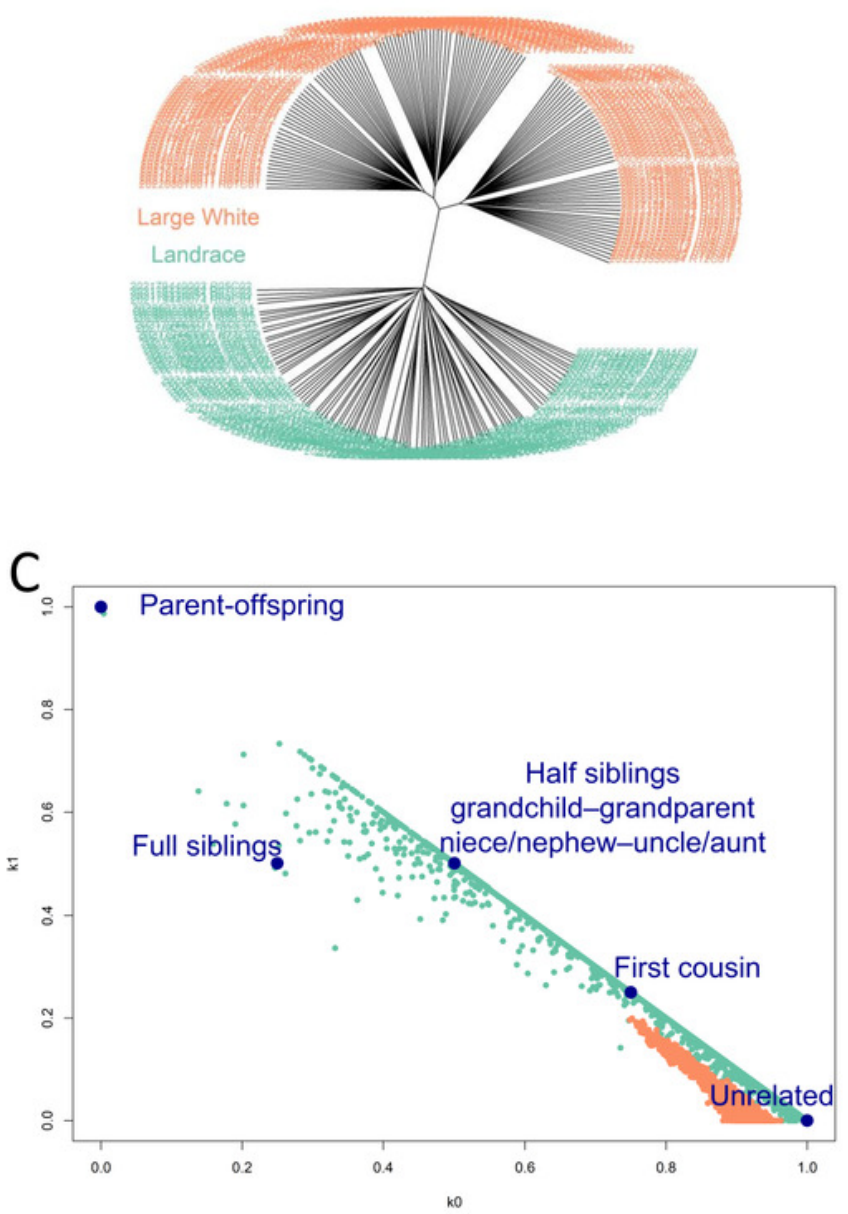

B

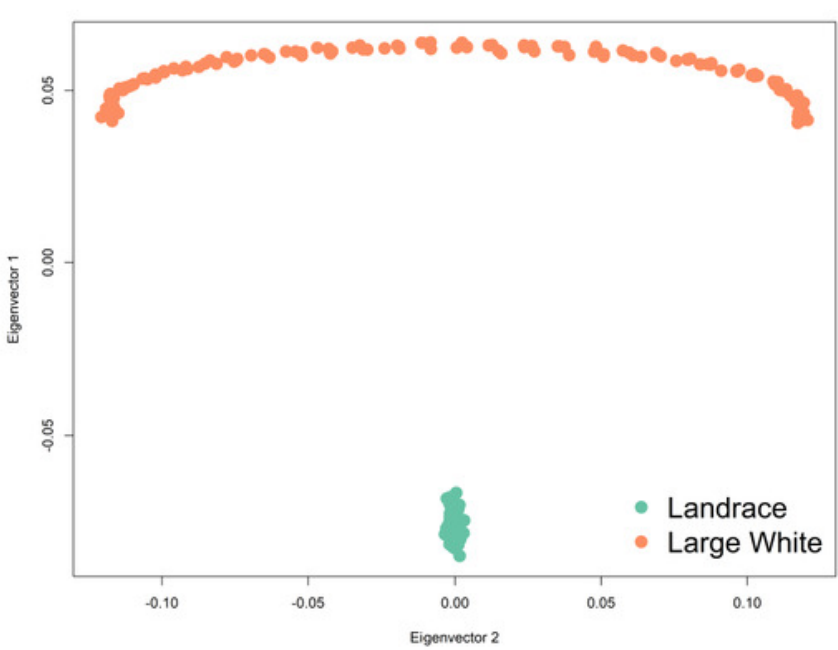

D

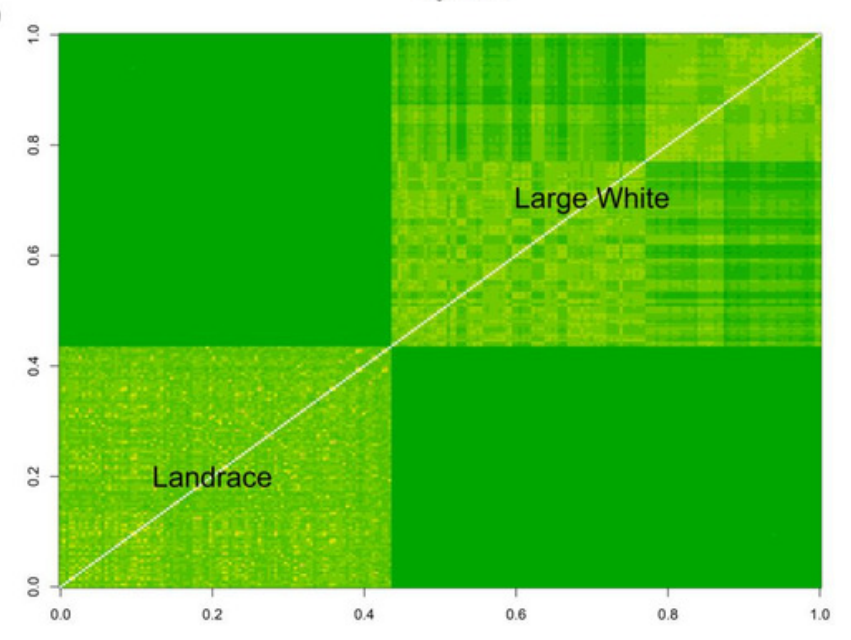




\section{Figure 3}

Manhattan plots

Manhattan plots for Splay Leg $(A, B, E, F)$ and Other Congenital malformations $(C, D, G, H)$ phenotypes in Landrace $(A, B, C, D)$ and Large White $(E, F, G, H)$ piglets, rrBLUP and Plink. Since Plink performs five tests for each SNP, the average negative logarithm of the $p$-value is plotted for Plink.

A
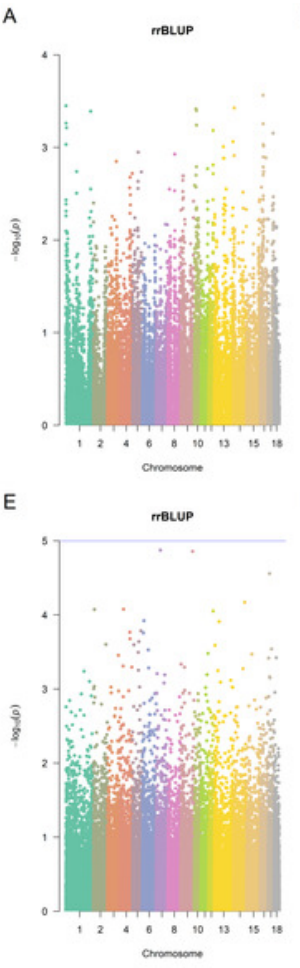
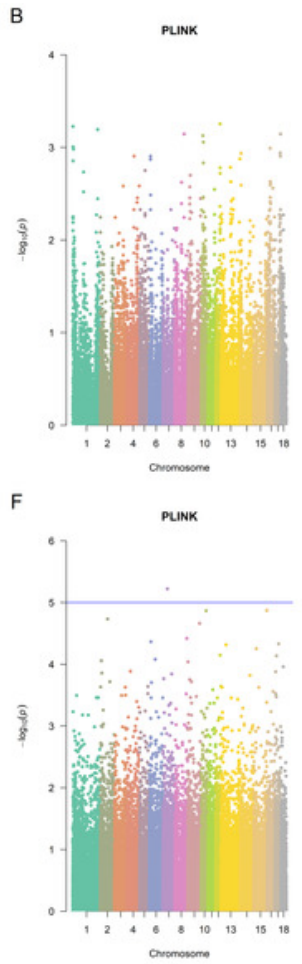
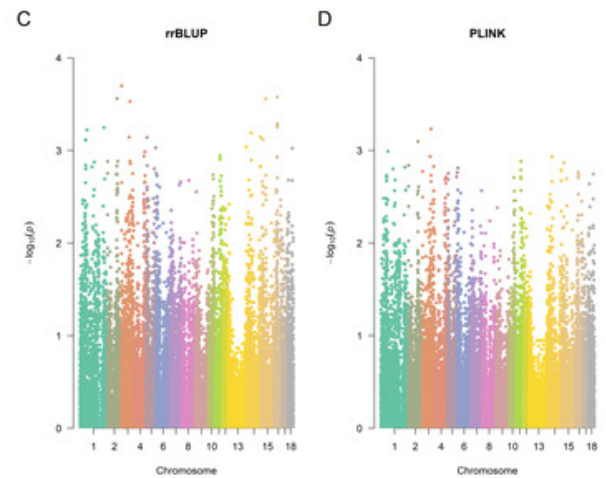

G

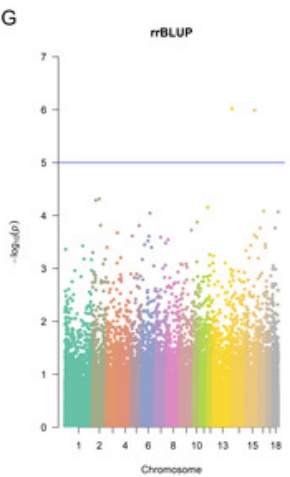

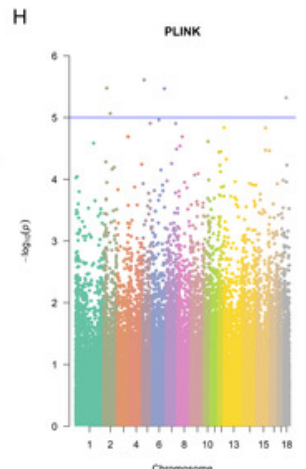

